\title{
Three-dimensional Organization of Lymphatics in the Dog Small Intestine: A Scanning Electron Microscopic Study on Corrosion Casts
}

\author{
Yasuaki Yamanaka, Keijiro Araki and Takuro Ogata \\ Department of Surgery, Kochi Medical School, Nankoku, Kochi, Japan
}

Received March 22, 1995

\begin{abstract}
Summary. Casts of the lymphatics of the canine small intestine were made by direct injection of a low viscosity methacrylate resin into a large lymphatic in the submucosal layer, and observed under a scanning electron microscope. The lymphatics started with rod-like central lacteals in the villi of the jejunum and leaf-like ones in those of the ileum. The bases of the lacteals were connected by slender lymphatics forming a threedimensional network, tentatively called the "superficial lamina propria lymphatic plexus". From this plexus, a few straight branches descended through the lamina propria to drain into a well developed "deep lamina propria lymphatic plexus", which was extended twodimensionally closely above the lamina muscularis mucosae. From this plexus, a few short lymphatics extended and penetrated the muscularis mucosae and drained into the "superficial submucosal lymphatic plexus", a coarse mesh work of thick lymphatics. From this plexus, a few slender lymphatics descended to drain into a second lymphatic plexus, called the "deep submucosal lymphatic plexus", which extended twodimensionally on the circular muscle layer. This deep submucosal plexus was a coarse network of thick knotty lymphatics. A large collecting lymphatic was occasionally seen running through the mesh. The tunica muscularis contained tubular lymphatics extending horizontally parallel to the muscle fiber, both circular and longitudinal.
\end{abstract}

The organization of lymphatics in the canine small intestine has been studied by various methods, such as Berlin blue injection into the lymphatic (SHImizu, 1932), India ink (VAJDA and TöMBÖL, 1964) or India ink injection into the parenchyma plus microangiography (SATOMURA et al., 1978). However, these studies used light microscopy with its limited depth of field, and the three-dimensional organization of the lymphatic system has not been sufficiently clarified.

Recently, the injection replica method in combination with scanning electron microscopy (SEM) was applied to studies of rat gastric lymphatic systems (TAMURA et al., 1977) and rabbit lymph nodes (KUROKAWA and OGATA, 1980). Using this method, the three-dimensional organization of lymphatics in the small intestine of the rabbit and rat has been replicated by direct injection of Mercox resin into the intestinal parenchyma, to be observed by SEM (OHTANI and OHTSUKA, 1985; OHTANI, 1987). In the canine intestine, however, production of precise casts of lymphatics by resin injection into parenchyma was difficult. In the present study, Mercox resin was directly injected into a large lymphatic in the submucosal layer of the dog small intestine, and satisfactory casts of lymphatics extended in each layer could be obtained. A precise description of the casts will comprise the present report.

\section{MATERIALS AND METHODS}

Adult hybrid dogs of either sex weighing $10-15 \mathrm{~kg}$ were used. They were fed without any specific dietary restrictions. The animals were laparotomized under general anesthesia with an intravenous injection of pentobarbital sodium. To relax the tension of the muscularis mucosae of the small intestine, papaverine hydrochloride $(0.01 \mathrm{~g} / \mathrm{kg}$ body weight) was infused intravenously according to the method by SATOMURA et al. (1978). The intestine was then divided into $10 \mathrm{~cm}$ segments by ligation with silk threads and each segment was severed. Thereafter, the feeding mesenteric artery to each segment was cannulated with a 20 -gauge flexible needle. The iso- 
lated intestines were perfused with physiological saline via the cannula until the intestinal wall was decolorized, and large lymphatics approximately $300 \mu \mathrm{m}$ in diameter in the submucosal layer were identified under a dissection microscope.

Mercox resin (CL-2B, Dai-Nippon Ink Corp, Japan) was diluted to $50 \%$ (vol/vol) with monomeric methyl methacrylate to obtain a lower viscosity. A small amount of resin, $0.2-0.8 \mathrm{ml}$, was manually injected by a 29 -gauge needle $(0.33 \mathrm{~mm}$ in diameter) into the large submucosal lymphatics in the isolated intestinal segment using manual pressure. The segment of the intestine was then immersed in a water bath at $25^{\circ} \mathrm{C}$ for $24 \mathrm{~h}$ to polymerize the resin. After the resin hardened, the whole segment was immersed in $20 \%$ $\mathrm{KOH}$ solution for $72 \mathrm{~h}$ to remove the tissue elements around the casts. The casts were washed with running water for $24 \mathrm{~h}$ and air-dried. They were mounted on metal stubs, and coated with gold in an ion coater (Eiko IB-5; Eiko Engineering, Tokyo) to be observed under an SEM, Hitachi S-450.

\section{RESULTS}

\section{Lymphatics in the mucosal layer}

The lymphatic canal of the canine jejunum started with a single central lacteal of a rod-like shape, approximately $1,100 \mu \mathrm{m}$ in height and $120 \mu \mathrm{m}$ in maximum caliber (Fig. 1a). The caliber of the upper one fourth of the lacteal was slightly larger than that of the lower three fourths. Under higher magnification, several circular constrictions were seen near the top (Fig. 1b). In addition, oval indentations corresponding to the impressions by the endothelial nuclei were seen. The casts of the central lacteals of the canine ileum were slender leaf-like shapes, being compressed in an axial direction. They were slightly lower in height than the jejunal lacteals, which were tubular in shape (Fig. 2). Except for these minor differences in the central lacteals, the basic structures of the lymphatic casts in each layer were similar between these two areas of the small intestine. Therefore, observations in the ileum are described in this paper.
From the bottom of the central lacteals, some slender lymphatics were emitted and ran in various directions, forming a three-dimensional network around the base of the lacteals (Fig. 3). This plexus is tentatively called the "superficial lamina propria lymphatic plexus" in this paper.

From this plexus, a few slender connecting lymphatics divided, descended through the lamina propria without ramification, and drained into the lymphatic plexus located immediately above the muscularis mucosae (Fig. 4). This plexus was a twodimensional network consisting of slender lymphatics approximately $50-100 \mu \mathrm{m}$ in caliber and tentatively called the "deep lamina propria lymphatic plexus" (Figs. 4-8). From this plexus, a few short lymphatics arose and penetrated through the thin layer of the muscularis mucosae, to be drained into the plexus of the superficial layer of the submucosa (Fig. 6).

\section{Lymphatics in the submucosal layer}

Two types of lymphatic plexuses were seen in the submucosal layer (Figs. 7-10). One type of plexus was a two-dimensional network $300-500 \mu \mathrm{m}$ in mesh size formed by thick lymphatics $30-70 \mu \mathrm{m}$ in caliber located immediately below the muscularis mucosae (Fig. 7). This plexus is here tentatively called the "superficial submucosal lymphatic plexus". From this plexus, a few slender lymphatics divided, ran downward and drained into the lymphatic plexus situated closely above the circular muscle layer (Fig. 9). This second lymphatic plexus was a two-dimensional network $200-300 \mu \mathrm{m}$ in mesh diameter formed by thick knotty lymphatics approximately $50-90 \mu \mathrm{m}$ in caliber showing a caterpillar-like appearance (Fig. 10). This plexus is called the "deep submucosal lymphatic plexus" in this paper.

A general luminal side view from the layer above the muscularis mucosae to the proper muscle layer is shown in Figure 11. Here can be seen a very large collecting lymphatic approximately $250 \mu \mathrm{m}$ in caliber. It originated from the superficial submucosal lymphatic plexus and was located between the two plexuses (Figs. 8, 11). Typical valvular structures were occasionally seen (Fig. 8).

Fig. 1. An SEM view of a corrosion cast of central lacteals in dog jejunum. a. Low-powered view showing rod-shaped lacteals. $\times 70$. b. A higher magnification of a part of Figure 1a, showing circular constrictions (arrow) near the top of the lacteals. Arrow-heads indicate imprints of endothelial nuclei. $\times 270$

Fig. 2. Central lacteals in the ileum, which are slender leaf-like forms. $\times 110$

Fig. 3. Lymphatic cast close to the base of the lacteals $(L)$. The lymphatics extending from the lacteals form a three-dimensional network, the superficial lamina propria lymphatic plexus $(S L) . \times 140$ 

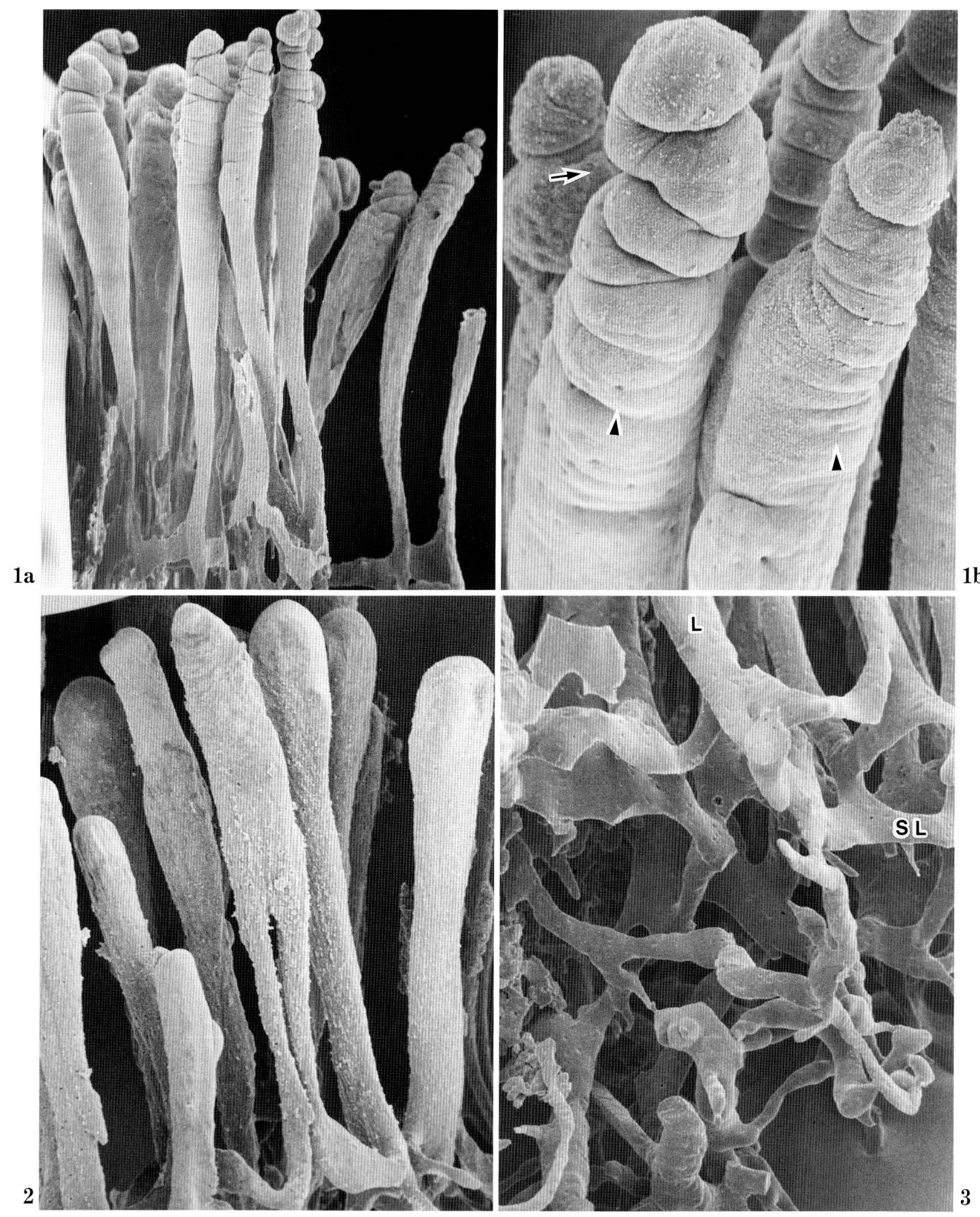

Figs. 1-3. Legends on the opposite page. 

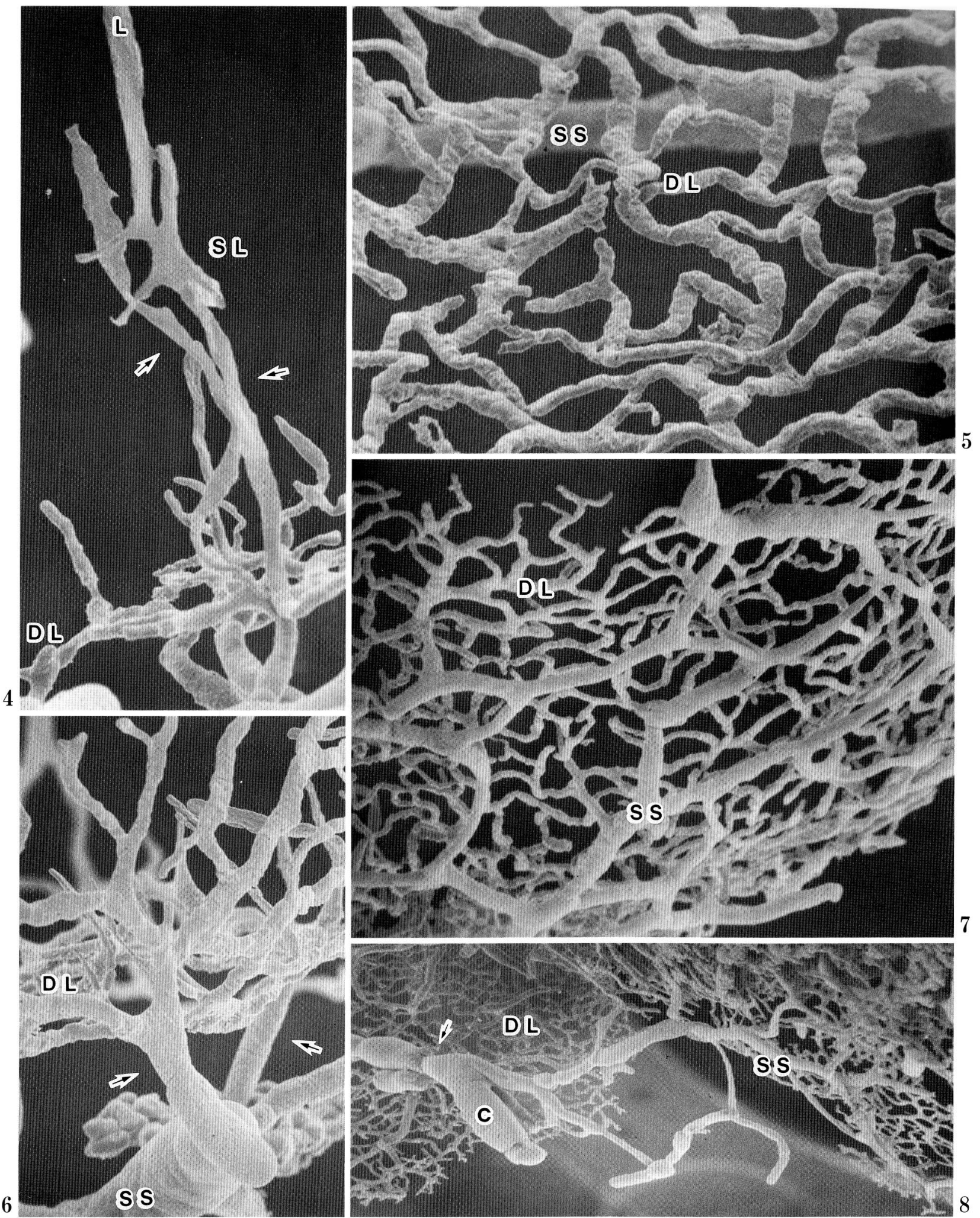

Figs. 4-8. Legends on the opposite page.

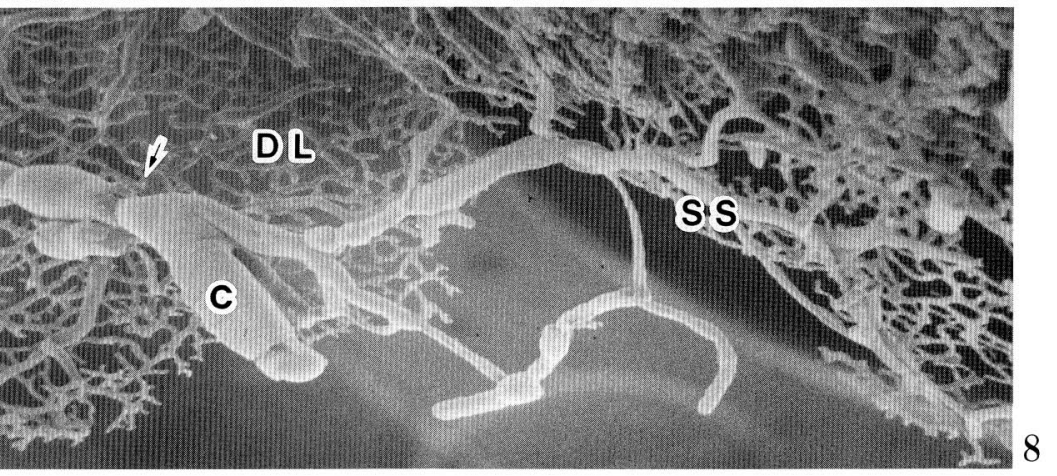



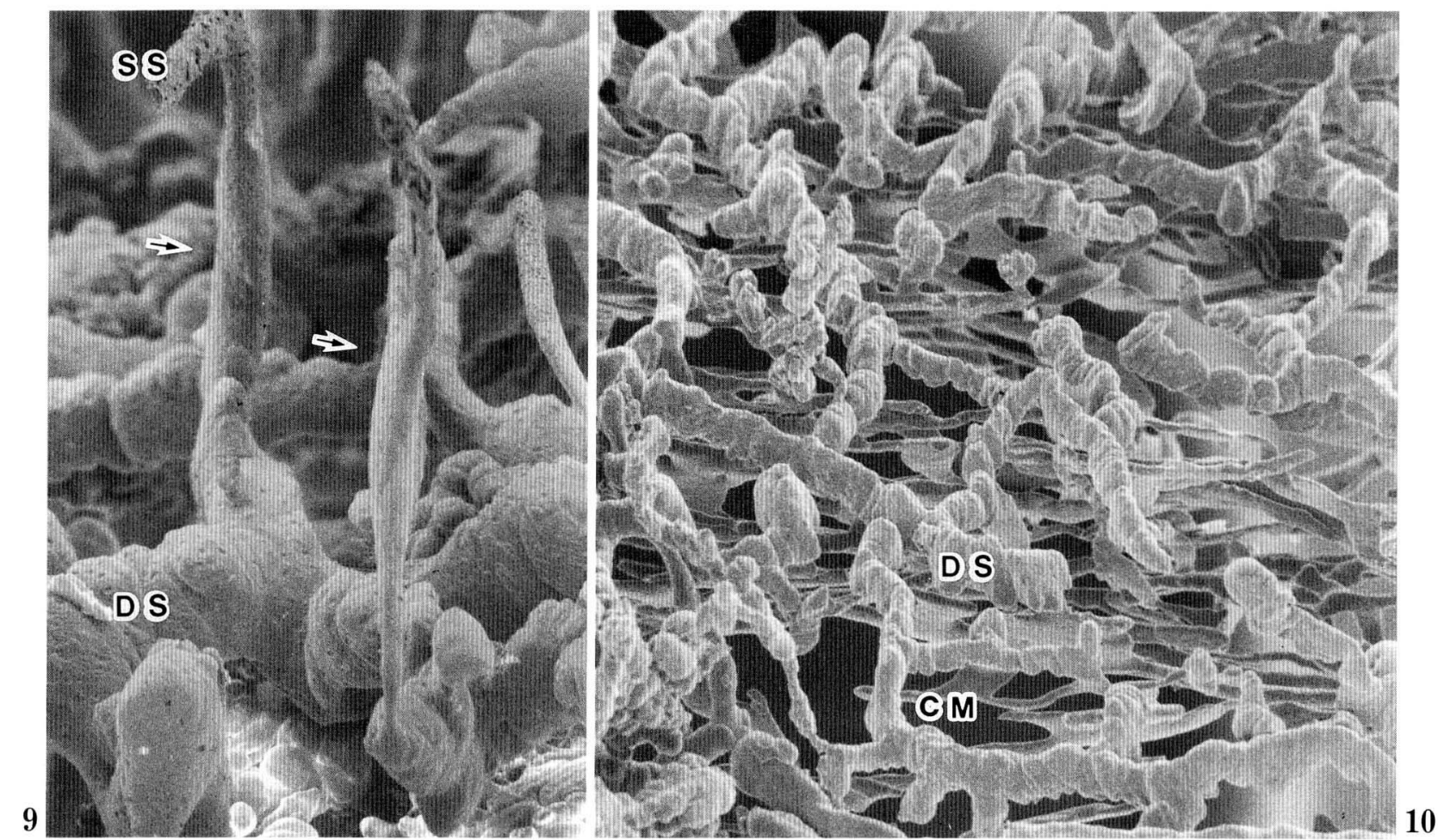

Fig. 9. The straight connecting lymphatics (arrows) drain into the deep submucosal lymphatic plexus (DS), taking on a caterpillar-like appearance. SS superficial submucosal lymphatic plexus. $\times 160$

Fig. 10. A luminal side view of the deep submucosal lymphatic plexus $(D S)$ which forms a coarse twodimensional network. Slender circular muscle lymphatics $(C M)$ can be seen through it. $\times 60$

\section{Lymphatics in the proper muscle layer}

From the deep submucosal lymphatic plexus, bandlike lymphatics were issued to enter the circular muscle layer (Fig. 12). In this layer, superficial lymphatics were band-shaped and usually ran obliquely toward the serosal side. They were gradually transformed into tubular-shaped lymphatics, which ran parallel to the circular muscle fibers (Figs. 12, 13). An oblique side view (Fig. 14) facilitated observation of the connection between the band-like and tubularshaped lymphatics. The tubular-shaped ones occasionally drained into the large collecting lymphatic (Fig. 15).

The lymphatics in the circular muscle layer drained into the lymphatics in the longitudinal muscle layer. In this muscle layer, they ran parallel to the longitudinal muscle fibers (Fig. 16), but were less developed than in the circular muscle layer. The

Fig. 4. Lymphatics (arrows) connecting the superficial lamina propria lymphatic plexus (SL) and the deep lamina propria lymphatic plexus $(D L) . \times 90$

Fig. 5. Deep lamina propria lymphatic plexus $(D L)$ forming a two-dimensional network of small meshes. Under the plexus, a superficial submucosal lymphatic (SS) can be seen. $\times 150$

Fig. 6. A serosal side view of the deep lamina propria lymphatic plexus (DL). Connecting lymphatics (arrows) extending from the plexus penetrate the muscularis mucosae to drain into the superficial submucosal lymphatic plexus (SS). $\times 120$

Fig. 7. A serosal side view of the superficial submucosal lymphatic plexus (SS) and the deep lamina propria lymphatic plexus $(D L) . \times 60$

Fig. 8. A serosal side view of a submucosal collecting lymphatic $(C)$. Note that the lymphatic arising from the superficial submucosal lymphatic plexus drains into a large collecting lymphatic. The constricted part of the cast (arrow) corresponds to the lymphatic valve. $\times 30$ 


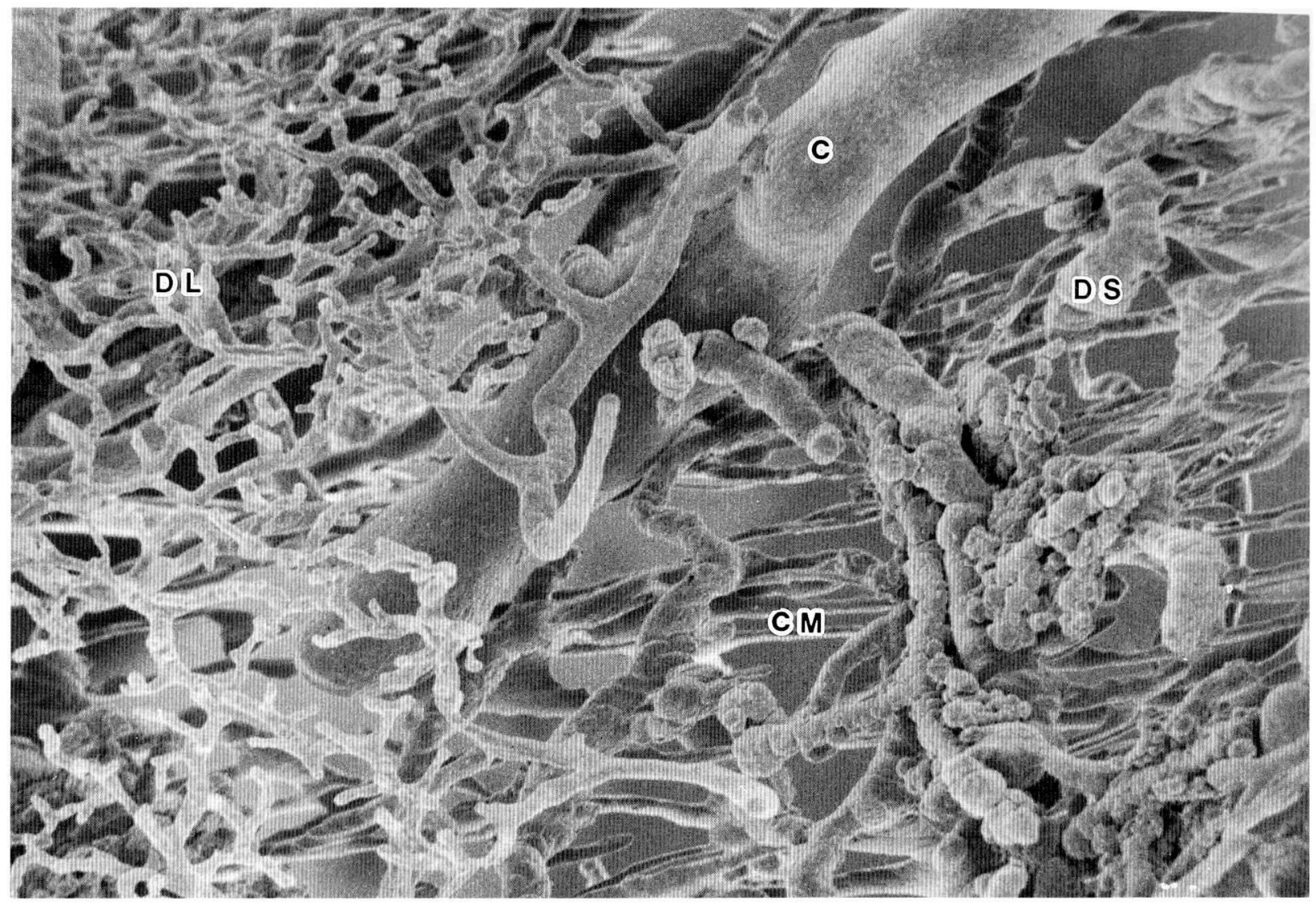

Fig. 11. A luminal side view of a lymphatic cast. In the left half of the picture, the deep lamina propria lymphatic plexus remains, but on removal, the deep submucosal lymphatic plexus (DS) and circular muscle lymphatics $(C M)$ are exposed in the right half. Note the large collecting lymphatic $(C)$ passing through these layers. $\times 80$

lymphatics of the tunica muscularis drained into the large efferent lymphatics (Fig. 18).

\section{Lymphatics in the subserosal layer}

In the subserosal space, a flat lymphatic approximately $50-100 \mu \mathrm{m}$ in thickness was seen. Typical lymphatic valves were seen (Fig. 17).

The casts of the efferent lymphatics were large and had successive V-shaped incisurae corresponding with valves (Fig. 18).

\section{DISCUSSION}

In previous studies of the lymphatic casts of murine small intestine (OHTANI, 1987), Mercox resin was directly injected into the parenchyma of the intestinal

Fig. 12. A lateral side view of the cast of the circular muscle lymphatic $(C M)$. Band-shaped lymphatics dividing from the deep submucosal plexus $(D S)$ run obliquely and parallel to the circular muscle fibers. Note that superficial lymphatics are band-shaped but gradually transform into tubular-shaped lymphatics toward the deep layer. $\times 60$

Fig. 13. Higher magnification of the tubular-shaped lymphatic casts in the deep layer of the circular muscle layer. Lymphatics mainly run horizontally. $\times 110$

Fig. 14. A luminal and oblique side view of a lymphatic cast in the circular muscle layer. Band-shaped and tubular-shaped lymphatics (arrow) are seen. $\times 60$

Fig. 15. A lymphatic in the circular muscle layer draining into a collecting lymphatic (arrow). $\times 40$

Fig. 16. A serosal side view of a cast showing lymphatics in the longitudinal muscle layer (arrows). $\times 60$ 


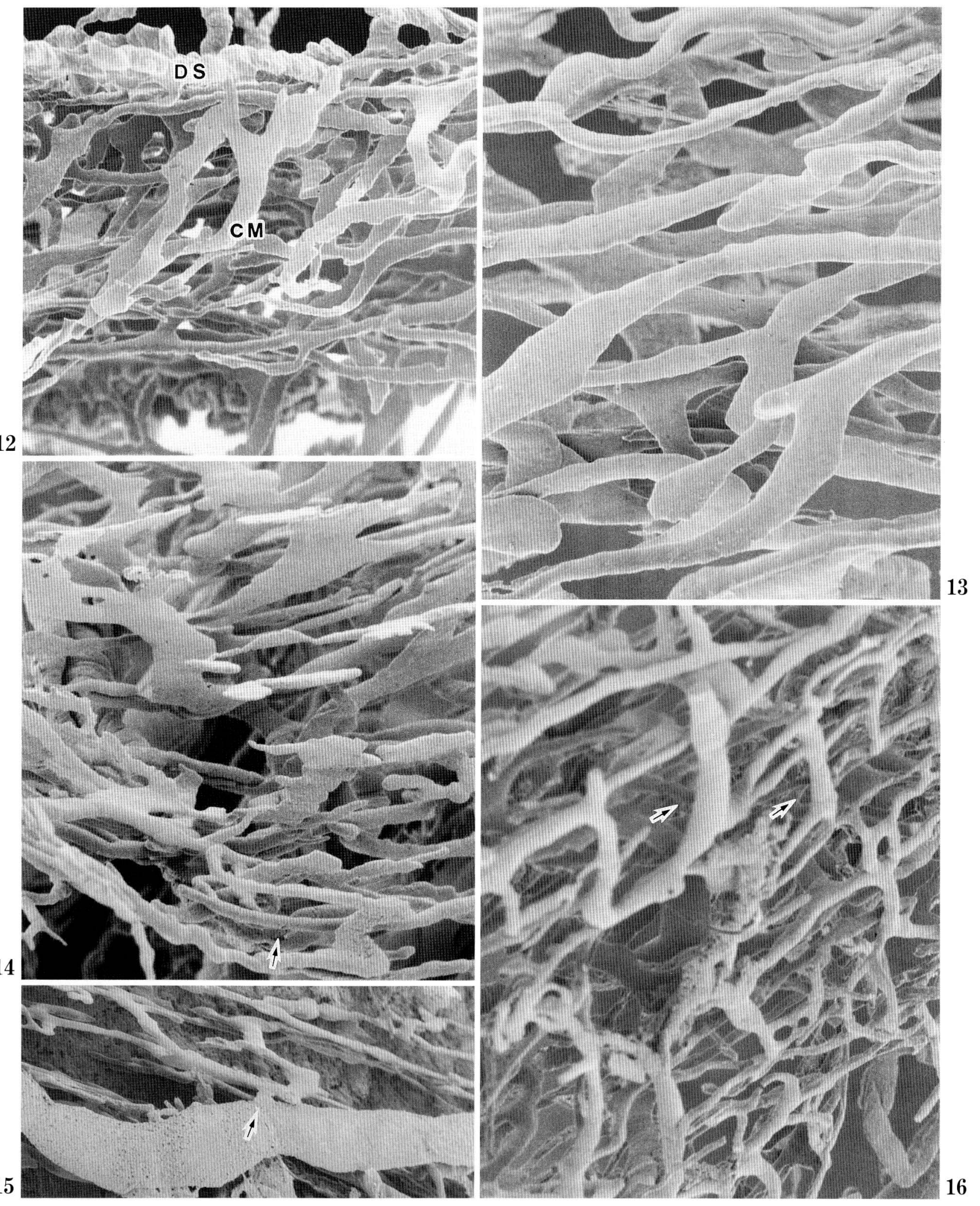

Figs. 12-16. Legends on the opposite page. 

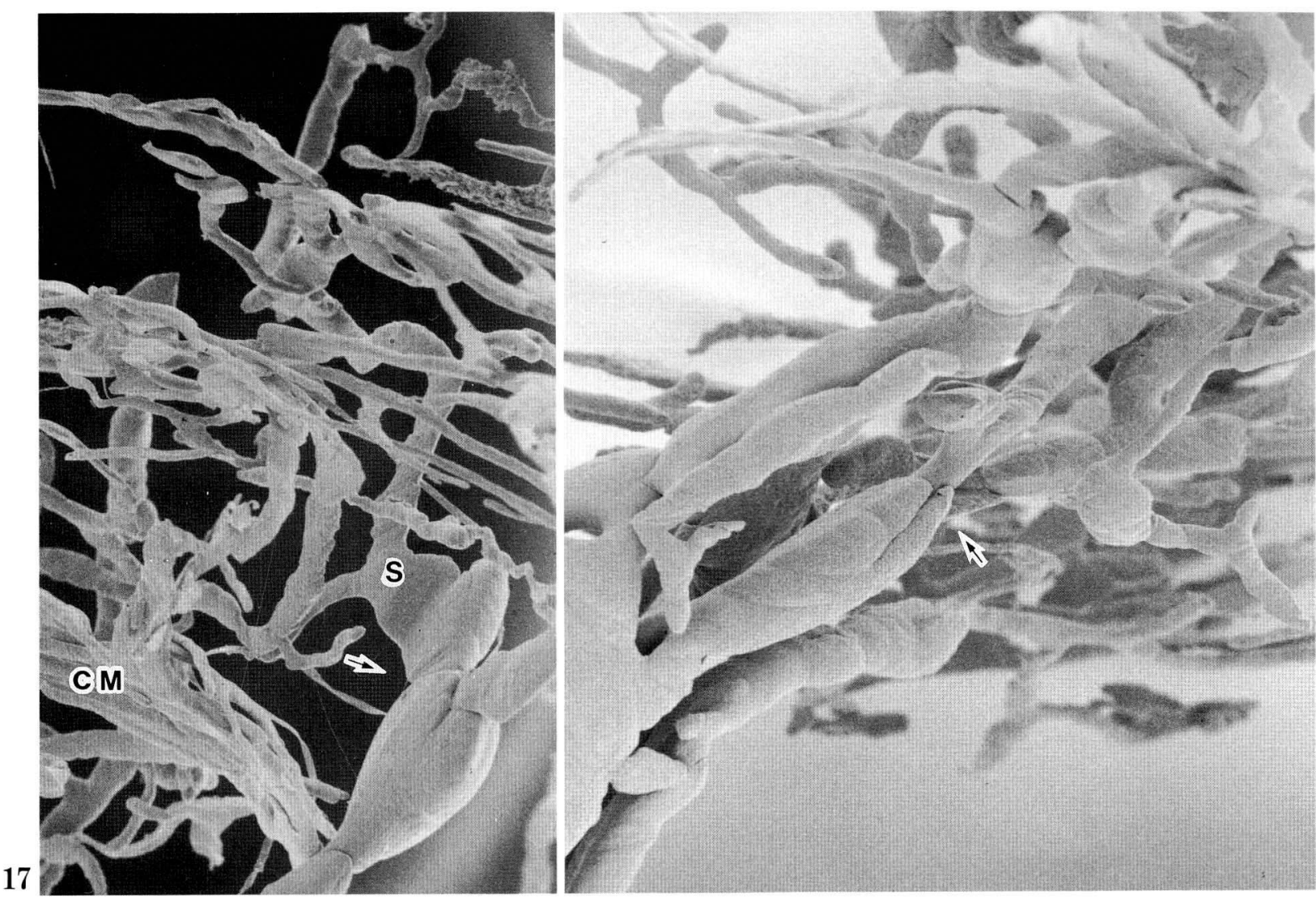

Fig. 17. A luminal side view of the subserosal lymphatic $(S)$ showing a typical valvular structure $($ arrow $) . \times 60$

Fig. 18. Cast of an efferent lymphatic. A deep V-shaped incisura corresponding with the valve (arrow) is seen. $\times 60$

wall. In the case of the canine intestine, however, it was difficult to obtain satisfactory lymphatic casts extended in each layer of the small intestine using this method, probably due to the lack of sufficient injection pressure. In addition, blood vessel casts, which were sometimes difficult to distinguish from the lymphatic casts, were concomitantly formed in the previous method. We devised a method using direct resin injection into a large lymphatic in the submucosal space under a dissection microscope and succeeded in obtaining casts free of concomitant blood vessel casts. However, a continuous lymphatic cast covering all the layers of the small intestine was not possible to produce, due to the presence of lymphatic valves. When the lymphatic space was amply filled with resin, it was frequently broken at the valves, just before the lymphatic space succeeding the valve was replicated. We therefore made many casts in repeated procedures until satisfactory ones in individual layers were obtained. By integrating the observations of those casts, the three-dimensional entity of the lymphatics in canine small intestine could successfully be visualized.

As is well known, the lymphatic canal of the small intestine begins at the central lacteal in the villus. The present observation of resin casts concurs with the early report by SHImizu (1932) in that the canine lacteal had a tubular appearance in the jejunum, while being compressed along the axial direction in the ileum.

It has been reported that the superficial lymphatic network connects the base of lacteals in the dog (VAJDA and TöMBÖL, 1964). Another report depicted the lacteals as united by arch-shaped lymphatics in the submucosal layer (JAMioLKowsKA, 1963). Using the India ink injection method, SATOMURA et al. (1978) showed two kinds of lymphatic networks in the lamina propria of the dog small intestine: the first was a horizontal lymphatic network situated at the bottom of the villi, and the second was a well developed horizontal network spreading immediately above the muscularis mucosae. In addition, they 


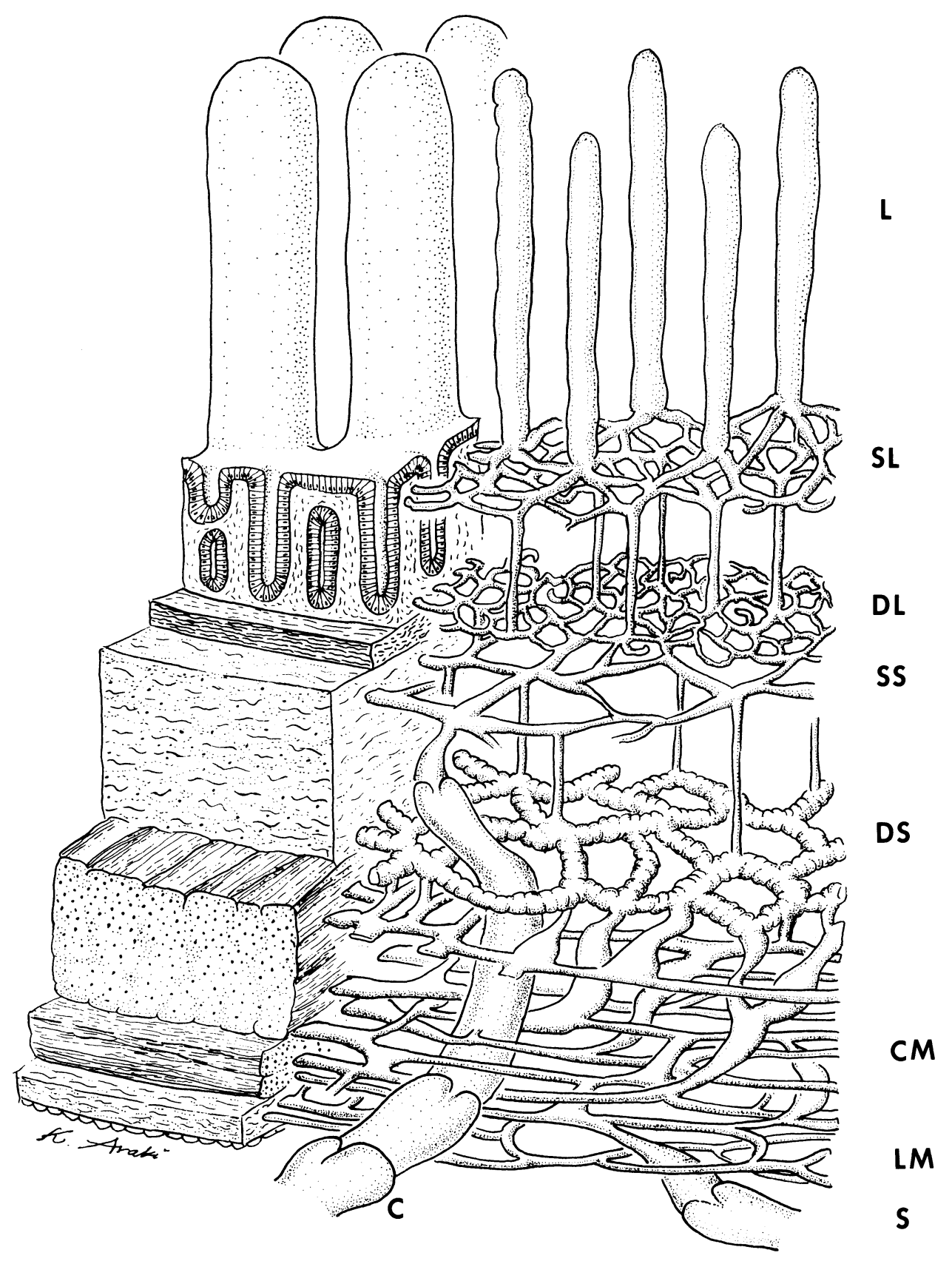

Fig. 19. A schematic representation of the three-dimensional organization of lymphatics in the canine ileum based on SEM observations of resin casts. $L$ lacteal, $S L$ superficial lamina propria lymphatic plexus, $D L$ deep lamina propria lymphatic plexus, $S S$ superficial submucosal lymphatic plexus, $D S$ deep submucosal lymphatic plexus, $C M$ circular muscle lymphatic, $L M$ longitudinal muscle lymphatic, $S$ subserosal lymphatic, $C$ collecting lymphatic. 
noted that straight branches from the first network traveled downward with no ramification and joined the second plexus. These lymphatic structures were more precisely and more convincingly demonstrated by the present cast study.

Lymphatics in the submucosal layer of the small intestine have been reported by several investigators (JAMIOLKOWSKA, 1963; VAJDA and TÖMBÖL, 1964; DoBBINS, 1966). However, no reports seem to have described the existence of two kinds of lymphatic networks in this layer, something clearly established in the present study. The first one, tentatively called the "superficial submucosal lymphatic plexus", spreads closely under the muscularis mucosae to form a coarse network of thick lymphatics. The second one, calld the "deep submucosal lymphatic plexus", located closely above the circular muscle layer, comprises knotty lymphatics of a caterpillarlike appearance. Furthermore, a few slender connecting lymphatics as well as a very large collecting lymphatic ( $300 \mu \mathrm{m}$ in caliber) were noted. The latter lymphatic in the canine small intestine was previously described by SATOMURA et al. (1978).

Previous researchers reported that most lymphatics in the proper muscle layer ran parallel to the muscle fibers both in the circular and longitudinal muscle layer (VADJA and TÖMBÖL, 1964: UNTHANK and BoHLEn, 1988). Our cast observations, on the other hand, showed that the lymphatics in the circular muscle layer formed a band-like shape and gradually transformed into tubular-shaped lymphatics toward the longitudinal muscle layer. These bandlike shapes of the lymphatics may be ascribed to the narrow spaces among the circular muscle fibers.

Regarding the lymphatic valves, DoBBINS (1966) noted that they first appear in the submucosal layer. The present study verified that the cast with a typical valvular structure was first recognized in the lymphatics of the submucosal layer.

To summarize our results, the three-dimensional organization of the lymphatics in the canine ileum is schematically represented in Figure 19.

Acknowledgements. The authors are grateful to Mr. Y. YAMASAKI, Ms. K. IKEDA, M. MiYATA, M. InOUE and N. RoKUBO for their technical assistance.

\section{REFERENCES}

DobBins, W. O.: The intestinal mucosal lymphatic in man. A light and electron microscopic study. Gastroenterology 51: 994-1003 (1966).

Jamiolkowska, K.: The lymphatic vessels of the small intestine. Fol. Morphol. 22: 123-144 (1963).

Kurokawa, T. and T. Ogata: A scanning electron microscopic study on the lymphatic microcirculation of the rabbit mesenteric lymph node. A corrosion cast study. Acta Anat. 107: 439-466 (1980).

Ohtani, 0.: Three-dimensional organization of lymphatics and its relationship to blood vessels in rat small intestine. Cell Tiss. Res. 248: 365-374 (1987).

Ohtani, O. and A. OHtsuka: Three-dimensional organization of lymphatics and their relationship to blood vessels in rabbit small intestine. A scanning electron microscopic study of corrosion casts. Arch. Histol. Jap. 48: 255-268 (1985).

Satomura, K., N. TANigaWa and S. Magari: Microlymphangiographic study of lymphatic regeneration following intestinal anastomosis. Surg. Gynecol. Obstet. 146: 415418 (1978).

Shimızu, S.: Darmzotten und ihre Gefäße, insbesondere die Chylusgefäße der Säugetiere und des Menschen. Fol. Anat. Jap. 10: 193-227 (1932).

Tamura, A., T. Kurokawa, S. Yamada, M. Nishiyama, T. Sakamoto, R. Sato, S. Kinoshita, N. Chikasawa, Y. Yoshil, S. Takata, S. Tamura and T. Ogata: Scanning electron microscopic studies on the lymphatic vessels of the rat stomach. J. Clin. Electron Microsc. 10: 426-427 (1977).

UNTHANK, J. L. and H. G. BoHLEN: Lymphatic pathways and role of valves in lymph propulsion from small intestine. Amer. J. Physiol. 254 (Gastrointest. Liver Physiol. 17): G389-G398 (1988).

VAJDA, J. and T. TömвöL: Die Lymphgefäßstruktur der Dünndarmwand. Acta Morphol. Acad. Sci. Hung. 13: 339-347 (1964).

山中 康明

783 高知県南国市岡豊町小蓮

高知医科大学

第一外科教室

Dr. Yasuaki YAMANAKA

Department of Surgery

Kochi Medical School

Nankoku, Kochi

783 Japan 